\title{
Supporting information: Achieving Circularly Polarized Surface Emitting Perovskite Microlasers with All-Dielectric Metasurfaces
}

\author{
Wei Dai ${ }^{1, \dagger}$, Yujie Wang ${ }^{1, \dagger}$, Ruixue ${ }^{1,+}$, Yubin Fan ${ }^{1}$, Geyang $Q u^{1}$, Yunkai Wu ${ }^{1}$, \\ Qinghai Song ${ }^{1,2}$, Jiecai Han ${ }^{3}$, Shumin Xiao ${ }^{1,2,3, *}$
}

1. Ministry of Industry and Information Technology Key Lab of Micro-Nano Optoelectronic Information System, Harbin Institute of Technology (Shenzhen), Shenzhen, 518055, China.

2. Collaborative Innovation Center of Extreme Optics, Shanxi University, Taiyuan 030006 China.

3. National Key Laboratory of Science and Technology on Advanced Composites in Special Environments, Harbin Institute of Technology, Harbin 150080, P. R. China. $\dagger$ These authors contribute equally to this work.

Emails: *shumin.xiao@,hit.edu.cn

In the main text, highly directional laser emissions with purely circularly polarization have been experimentally demonstrated. Here we show all the details about the design of $\mathrm{TiO}_{2}$ metalens and the experimental setup for optical measurements. The profile of focal spot of the metalens has also been characterized and shown in the supplemental information. 


\section{The design of experiment}

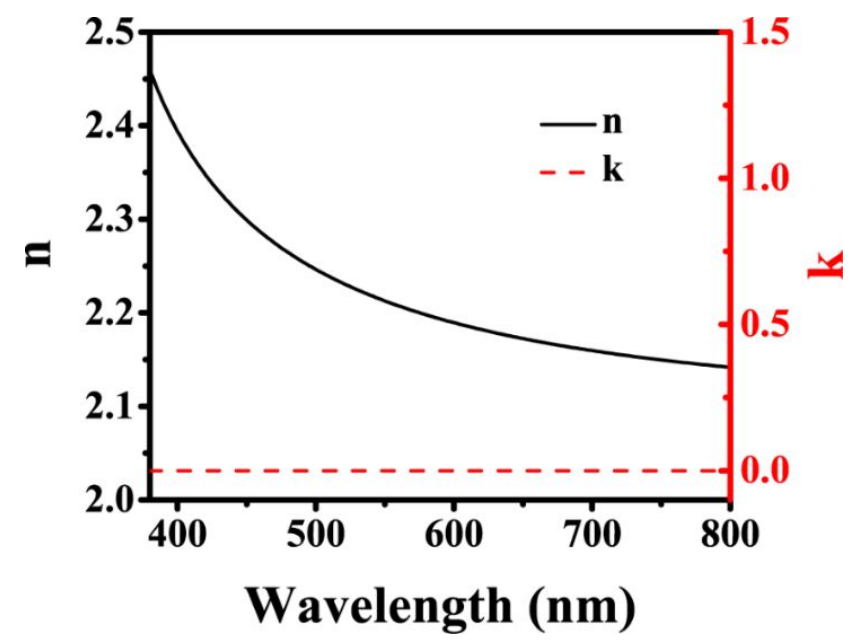

Figure S1: The refractive index (n) and light extinction coefficient (k) of TiO2 film.

In the main text, we have designed the rectangle shaped nanopillar for the geometrical phase based metalens. Here we show the detail about the optimization. The transmission and phase retardation between long and short axis have been numerically calculated as a function of $\mathrm{L}$ and $\mathrm{W}$ of the nanorod. The refractive index and the light extinction of $\mathrm{TiO}_{2}$ are taken from the experimental data shown in Fig. S1. The calculated results are depicted in Fig. S2. We can see that the nanorod with $\mathrm{L}=270 \mathrm{~nm}$ and $\mathrm{W}=60 \mathrm{~nm}$ can produce near unit transmission and the $\pi$ phase retardation. This is the fundamental building block of our metalens in the main text.
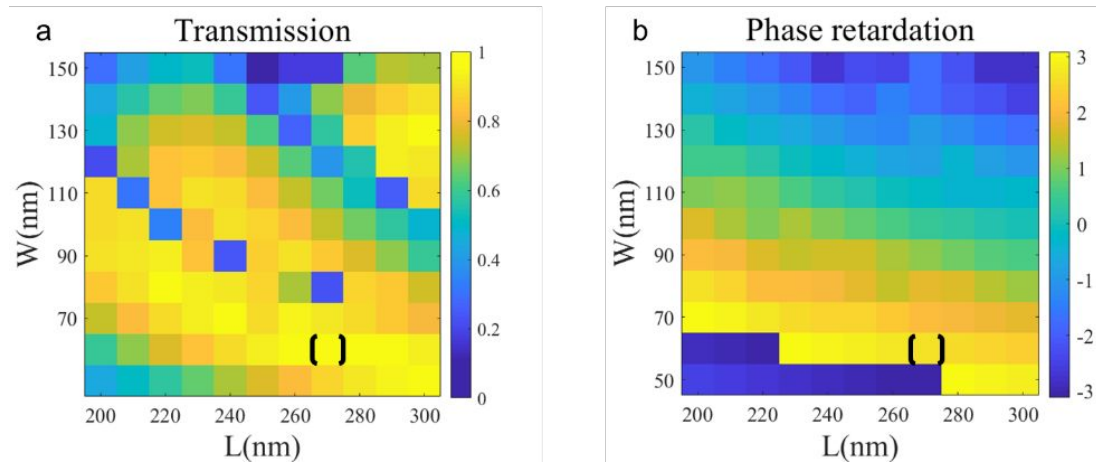

Figure S2: Optimization results for nanorod. (a) The transmission of nanorod with different $w$ and $l$. (b) The phase retardation of nanorod with different $w$ and $l$. 


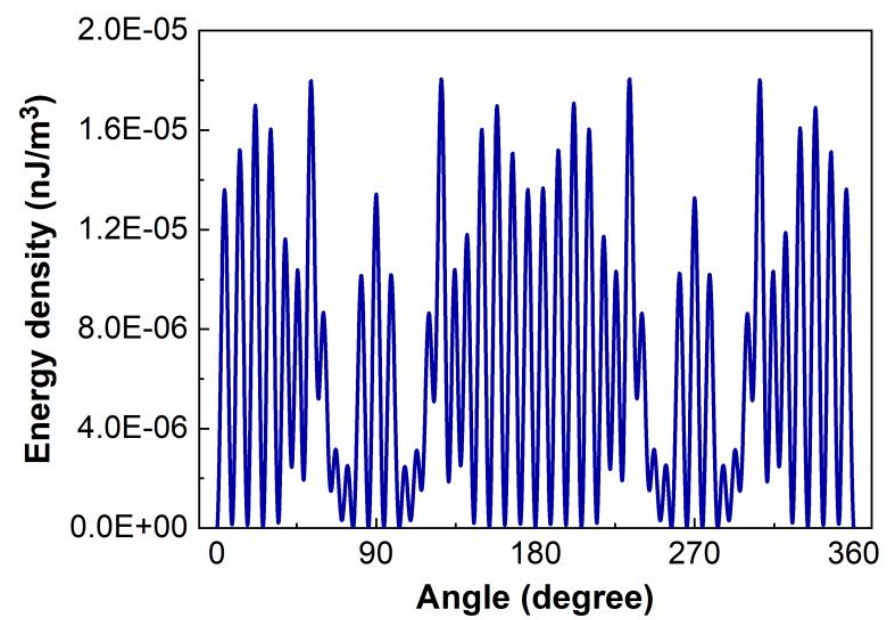

Figure S3: Far field energy distribution of the perovskite crystal.

For the optical experiment, the emissions from the perovskite microwire is also important. Since the microwire is partially pumped, it can produce whispering gallery modes in the transverse plane. The detail Q factors have been plotted in the main text. Here we show the corresponding far field patterns. Due to the rectangle shape of crosssection, we can see that the main emissions along the short sides in vertical directions. Figure S3 shows that the divergent angle is about 90 degree. This is one important parameter to design a metalens to match it. However, a more reasonable approach it replacing it with another microwire with different aspect ratio, which can produce narrow far field laser beams. 


\section{The setups for optical characterization}

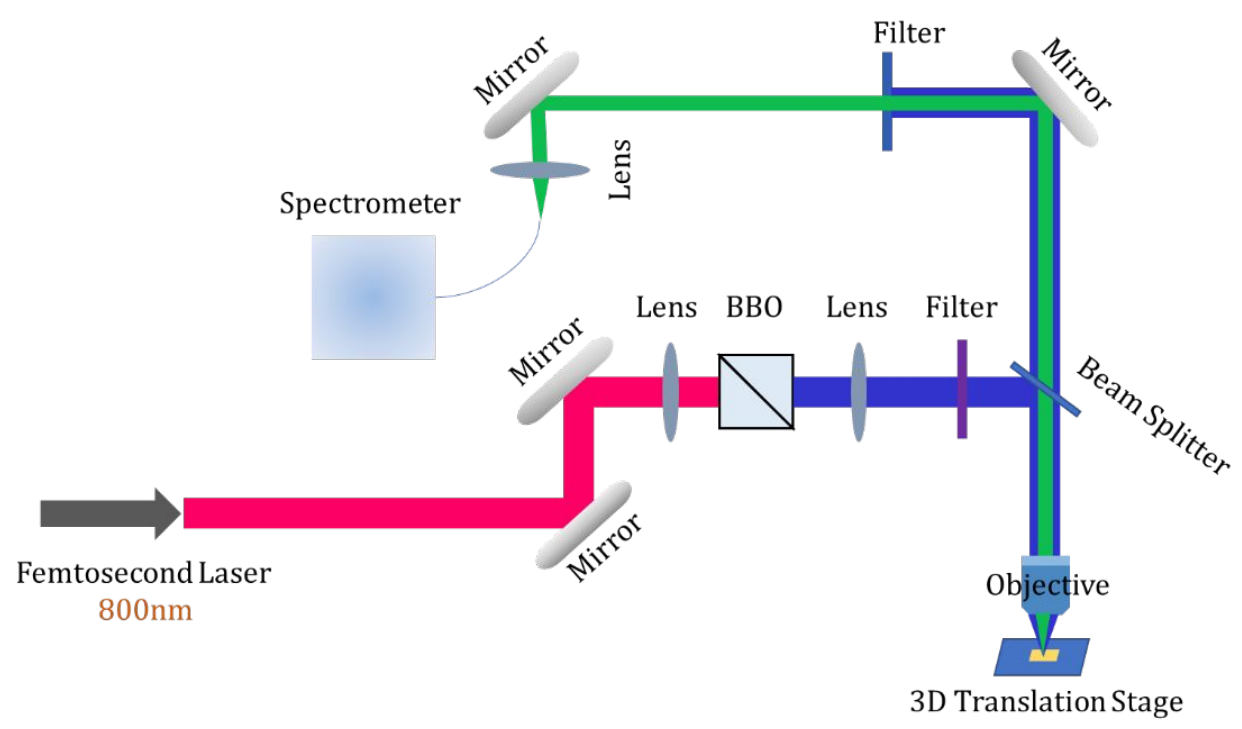

Figure S4. The home-made optical microscope for lasing measurement

Figure S4 shows the optical setup for measuring the laser characteristics from the perovskite microwire. The femtosecond laser at $800 \mathrm{~nm}$ was frequency doubled by a BBO crystal and focused by an objective lens onto the sample, which is placed onto a three-dimensional translation stage. The emitted light from the perovskite microwire is collected by the same metalens and collimated into a directional beam. The reflected laser is filtered out by a long-pass filter and the signals from the perovskite microwires are coupled into a CCD camera coupled spectrometer. Then the spectral information can be directly obtained. 


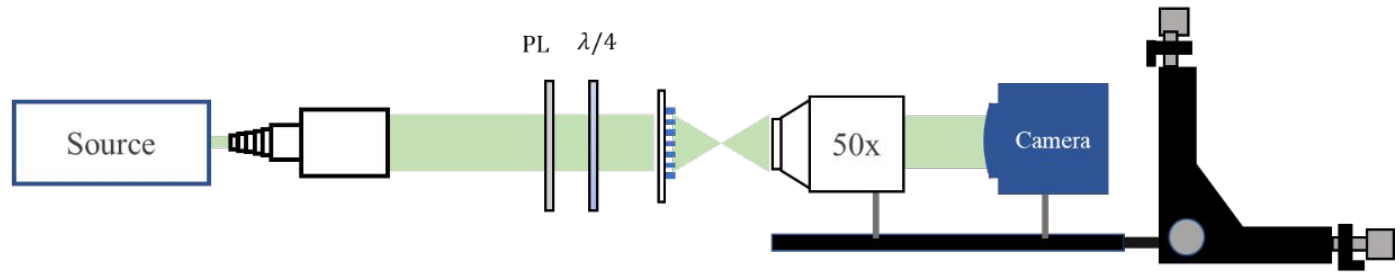

b

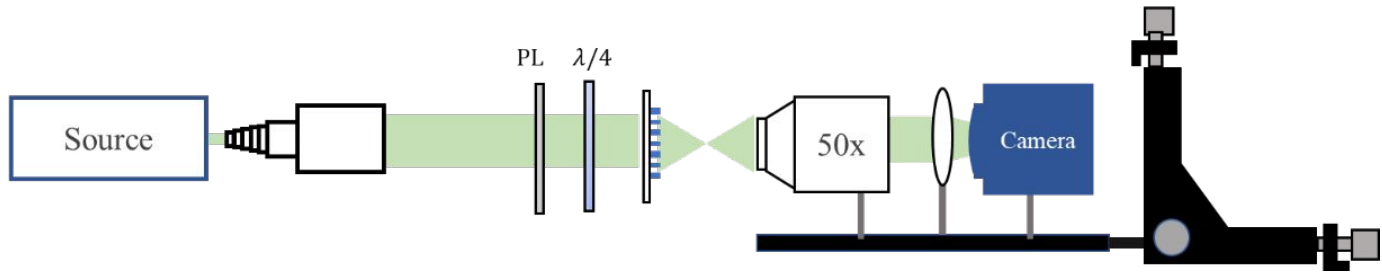

Figure S5: The optical setup for metalens characterization. (a) Focal spot measurement of metalens. (b) Focusing efficiency measurement of metalens.

Figure S5(a) shows the optical setup for the measurement of focal spots of the metalenses. It consists of a fiber-coupled light source, a linear polarizer, a quarterwaveplate, and an objective $(50 \times$ magnification, $\mathrm{NA}=0.55)$. The light from the source is converted to circular polarization and focused by the metalens. The focal spot is imaged by the objective lens and the CMOS camera. Figure S5(b) shows the optical setup to measure the focusing efficiency. The efficiency was directly obtained by comparing the collected signals with and without the metalens.

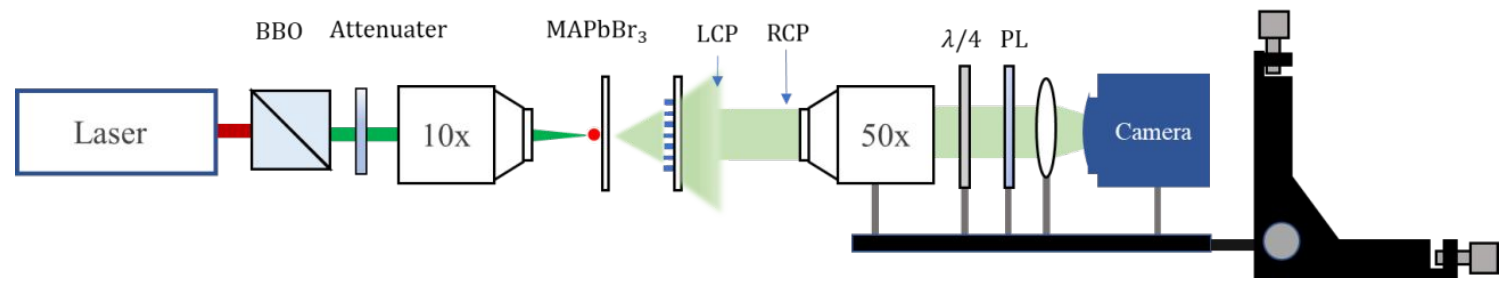

Figure S6: Measurement setup for lasing experiments.

Figure S6 shows the optical setup for measuring the directional emission of circularly polarized perovskite microlasers. It is a combination of Fig. S3 and Fig. S4. The incident light was focused by an objective lens onto the microwire. The emissions 
from the microwires was collected by the metalens. The corresponding polarization state, spectrum, and divergent angle were measured behind the metalens with a spectrometer or a CMOS camera.

\section{The recorded optical images}

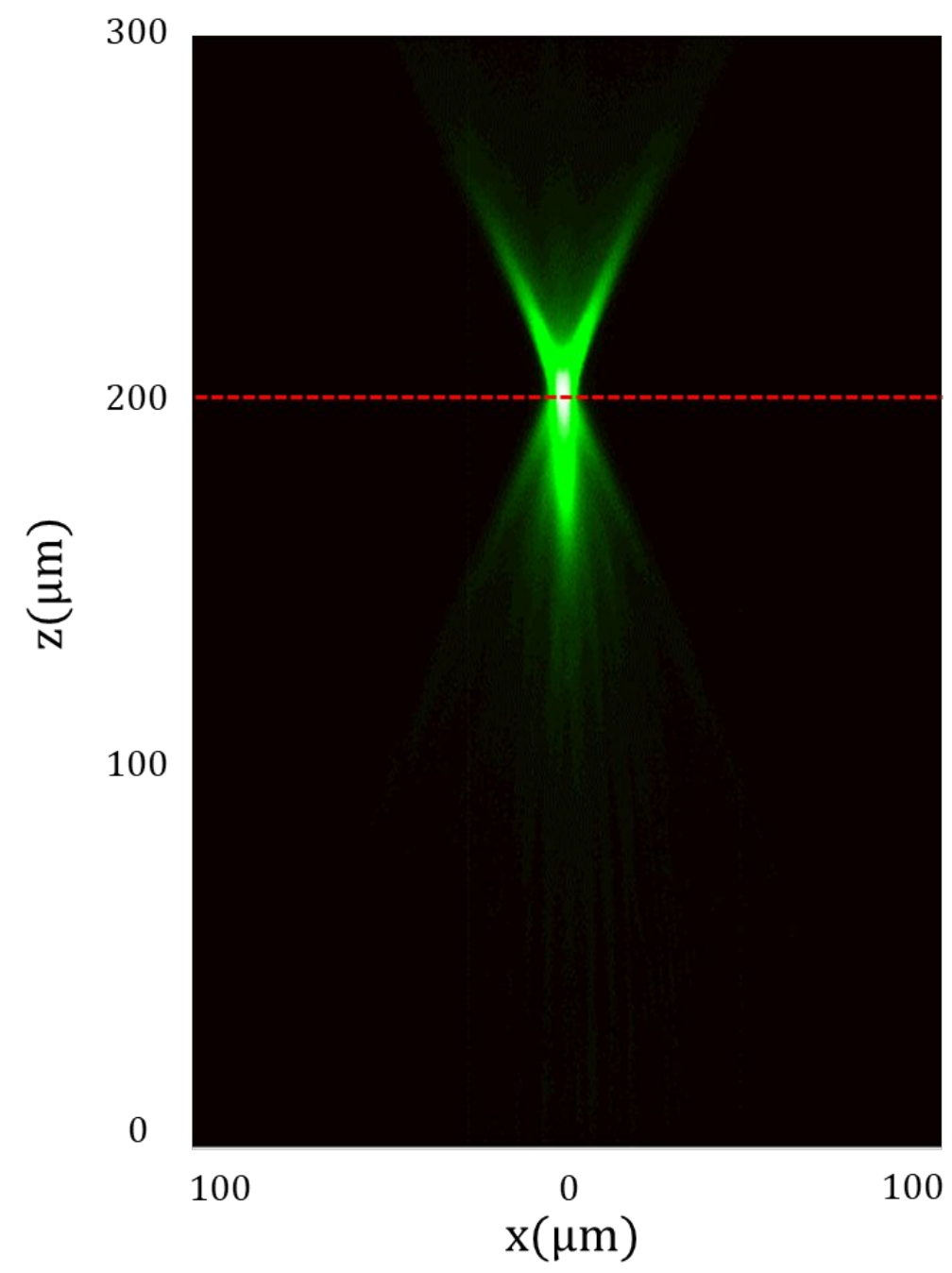

Figure S7. The experimentally recorded profile of the focal point in $x-z$ plane.

Figure S7 shows the profile of focus point in X-z plane. The incident laser at 520 nm was focused by the $\mathrm{TiO}_{2}$ metalens into a small focal spot. The profile in $\mathrm{x}-\mathrm{y}$ plane is shown in the inset of Fig. 2 in the main text. It is easy to see from Fig. S6 that the center of focal spot locates at $200 \mu \mathrm{m}$ away from the metalens, consistent with the 
numerical design very well. The calculated numerical aperture is NA $\sim 0.447$.

Figure S8 shows the intensity profile of left-handed circularly polarized laser beam recorded $2.213 \mathrm{~mm}$ behind the metalens. Here the intensity range of CCD was set the same as the one in Fig. 5 d. We can see that the left-handed circularly polarized light is almost the noise level and hard to seen. This is consistent with the divergent properties of metalens for the incident right-handed circularly polarized laser.

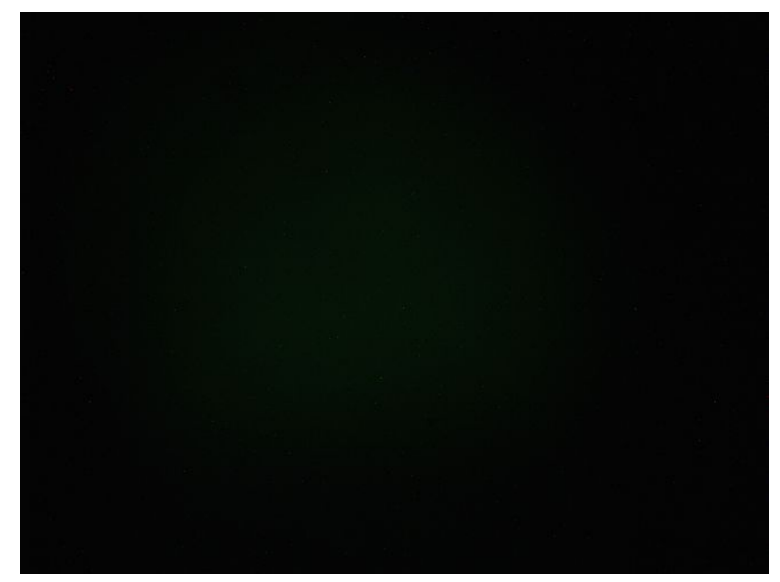

Figure S8: The LCP beam profile recorded at a position $2.213 \mu \mathrm{m}$ behind the $\mathrm{TiO}_{2}$ metalens

To determine the purity of the right-handed circular polarization, we have recorded the intensity profiles of two polarizations. The results are shown in Fig. S9. It is easy to see that the right-handed circular polarization is much stronger than the left-handed one. By integrated the intensity in the white circle and substrate the dark background, we find that the intensity ratio between two polarization is about $0.993: 0.007$. As a result, the total purity of the emitted laser recorded at $2.213 \mathrm{~mm}$ behind the metalens is about $99.3 \%$. This is consistent with the facts of collimation and divergence of two polarized light after passing the metalens. 

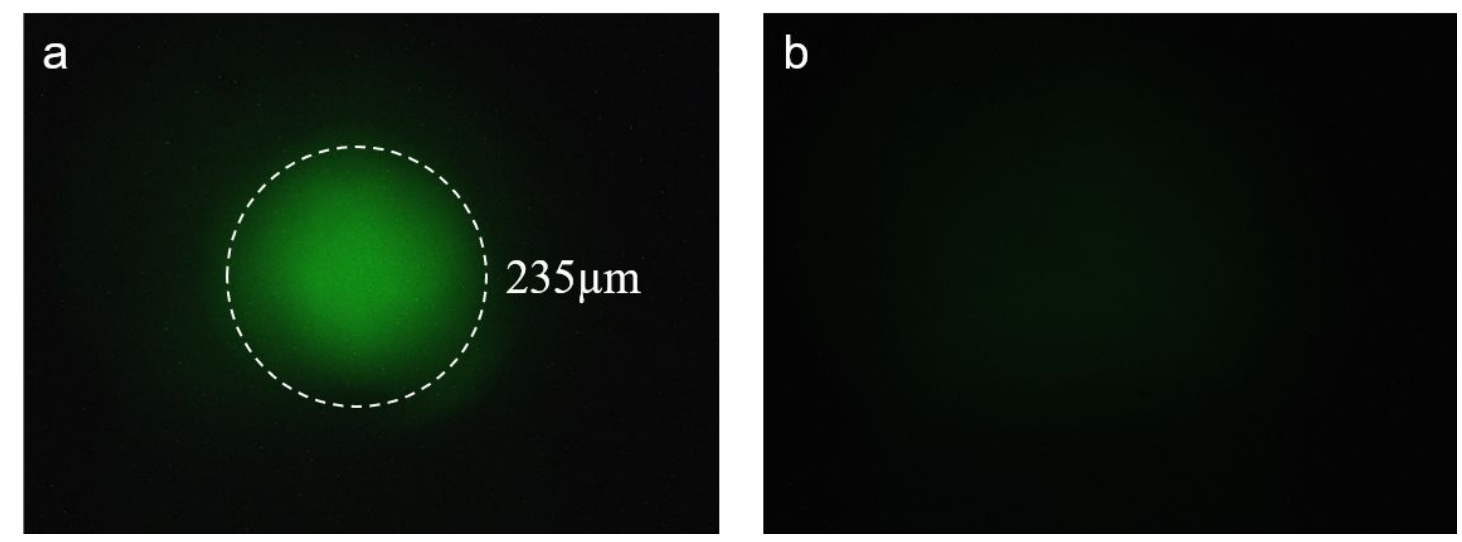

Figure S9: The surface emitting RCP and LCP perovskite laser. (a) The RCP beam profile recorded at a position $2.213 \mathrm{~mm}$ behind the TiO2 metalens. (b) The LCP beam profile recorded at a position $2.213 \mathrm{~mm}$ behind the $\mathrm{TiO} 2$ metalens.

\section{The further improvement}

In the main text, we have used the metalens with numerical aperture of 0.47 . This numerical aperture relates to a collection angle of $56^{\circ}$. Such a collection angle is smaller than the divergence angle of the laser emission from the microwire and thus wastes some emission energies. To increase the collection efficiency, one can either increase the numerical aperture of the metalens or reduce the divergence angle of microwire laser. Here we show the first possibility. Figure S10(b) shows the intensity profile along the diameter of the focal spot in $\mathrm{x}$ y plane. We can see that the incident laser at $520 \mathrm{~nm}$ is focused to a spot with full width at half maximum of $380 \mathrm{~nm}$. According to numerical aperture of 0.71 , this is very close to the diffraction limit. Figure S10(b) shows the wavelength dependent focusing efficiency. At the wavelength of laser emission, the efficiency is about $70 \%$. The large numerical aperture relates to a collection angle of 90.46 degree, larger than the divergence angle of laser emission. Therefore, the numerical aperture and the high efficiency can ensure the high efficiency collection and conversion of the perovskite microlaser to circularly polarized directional laser 
beam.

a

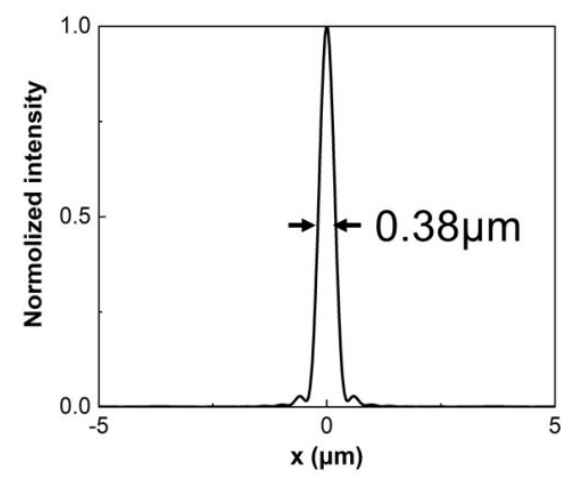

b

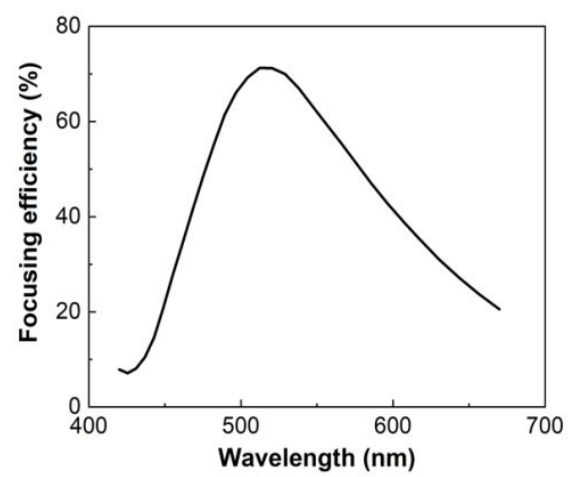

Figure S10: The numerical design of $\mathrm{TiO}_{2}$ metalens with numerical aspect of 0.71 . (a)

The intensity profile along the diameter of the focal spot. (b) The focusing efficiency of $\mathrm{TiO}_{2}$ metalens as a function of wavelength

\section{The repeated experiments}

In the main text, we show that the laser emission from a perovskite microwire can be converted to a circularly polarized beam with high directionality. To confirm the generality of this mechanism, we have replaced several different perovskite microwires and studied their laser emissions as well. Some of the experimental results are shown in Fig. S11 below. By replacing the microwire with a new one with a length of 23.554 micron, we have excited it with a frequency doubled Ti:Sapphire laser and recorded the far field beam 100 micron behind the metalens. The results are shown in Fig. S11(a). The central bright spot is right-handed circularly polarized. There is also a dim green background outside the central spot, which is caused by the divergence at the metalens. These phenomena are consistent with the results in main text very well. To further confirm it, we have replaced the third perovskite microlaser at the focal point of metalens. As shown in Fig. S11(b), the central bright spot is also right-handed circular polarized. 

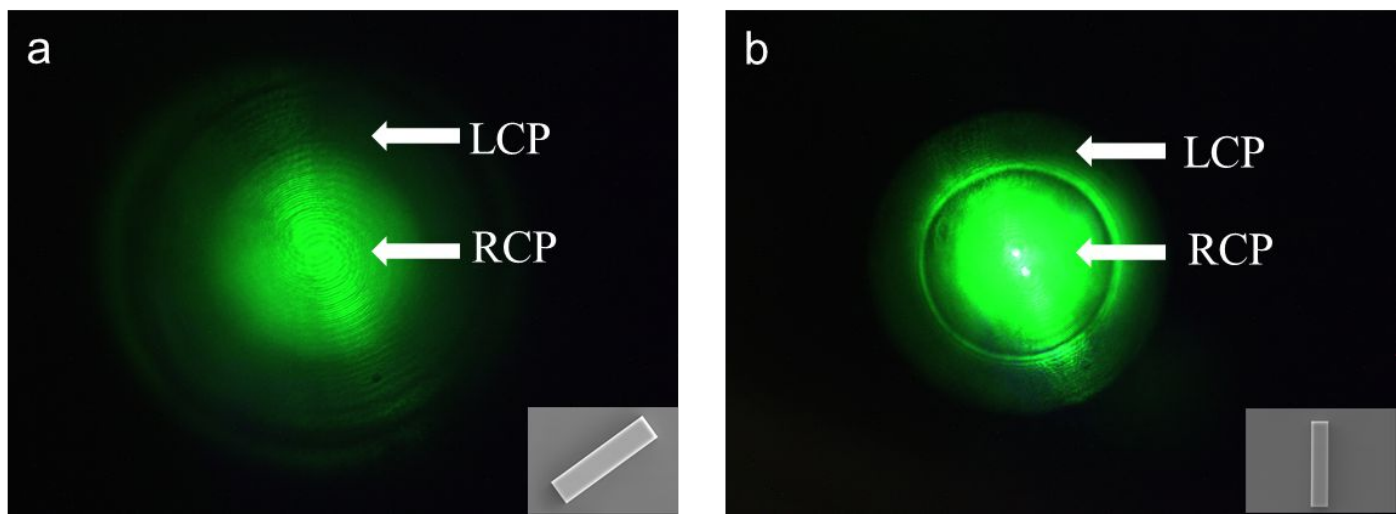

Figure S11. The repeated experiments with different perovskite microwire. (a) The far field beam profile of perovskite microlaser recorded $100 \mu \mathrm{m}$ behind the metalens for sample with length of $23.554 \mu \mathrm{m}$. (b) The far field beam profile of perovskite microlaser recorded $100 \mu \mathrm{m}$ behind the metalens for sample with length of $20.472 \mu \mathrm{m}$. 